\title{
Et la planète devint malade. Sur la montée des problèmes globaux et la généalogie du planétaire
}

\author{
Michel Damian \\ Économiste, Université de Grenoble, UMR 5194 PACTE, Pôle EDDEN, 38040 Grenoble, France
}

L'article qui suit a été rédigé il y a un quart de siècle, durant l'été et l'automne 1990. Il tentait, avec les limites des lectures de l'époque et, certainement, aussi avec quelque parti pris et quelques assertions empreintes du langage d'alors, de rendre compte de la généalogie du planétaire, du surgissement de la question du changement climatique et des problèmes globaux. Natures Sciences Sociétés a jugé bon de publier, dans la rubrique « Regards », cette contribution dans sa forme originelle (deux encadrés ont été ajoutés) ; elle apporte, en effet, des éléments historiques et contextuels précieux pour analyser la situation actuelle et elle montre bien la permanence ou le glissement de certaines questions.

Le contexte entremêlait les alertes des scientifiques sur la couche d'ozone et le climat, ainsi que la perception de ces périls inédits par les politiques et les grands médias; les débats entre scientifiques, physiciens et climatologues sur la théorie de « l'hiver nucléaire » et les conséquences planétaires de celle-ci ; les négociations autour de la "guerre des étoiles » et sur la diminution des arsenaux nucléaires entre les deux superpuissances ; la rhétorique de " La Terre, notre maison commune " par Mikhail Gorbatchev et la montée du concept de développement durable ; et, enfin, la guerre du Golfe et la géopolitique de l'énergie, comme un premier signe, ou un premier acte, d'embrasements à venir.

Encore aujourd'hui, à l'approche de la Conférence Paris Climat 2015, après les transformations engendrées par la globalisation économique et la prise de conscience des effets proprement géologiques des activités humaines (l'ère de l'Anthropocène, comme l'a nommée Paul Crutzen), l'archéologie du planétaire et de la montée des problèmes communs à l'humanité demeure, nous semble-t-il, un objet et un enjeu de connaissance.

Cet article a une histoire. Il avait été retenu, en décembre 1990, pour publication dans le numéro de février 1991 de la revue $L a$ Recherche. Il n'est cependant jamais paru. L'auteur dédie sa publication à Martine Barrère ; décédée en 1995, après avoir été chercheur au Commissariat à l'énergie atomique, puis enseignante à l'université, elle fut une grande figure du journalisme scientifique.

La Rédaction

La Terre fut carrée pour les Aztèques, ovale chez les Égyptiens, plate au Moyen Âge chrétien. Ce n'est plus aujourd'hui qu'une sphère malade, une petite boule en danger. De Washington à Moscou, de Paris à Londres, l'inquiétude gagne : la percée des problèmes environnementaux et des thèmes communs à l'humanité ne s'est pas démentie tout au long des années 1989 et 1990. La Terre parle, « comme Dieu prévenant Noé du Déluge », écrit l'éditorialiste de Time en janvier 1989.
Cette « intrusion soudaine et considérable » des problèmes d'environnement, pour reprendre les termes utilisés par le président François Mitterrand en juillet 1989 lors du sommet de l'Arche, réunissant les pays du G7, n'est pas simple à expliquer. Toujours est-il qu'un glissement s'est opéré. Vers un dérisoire consensus? Ou un nouvel habillage idéologique? Pas seulement.

La connexion des thèmes et la conjonction des craintes sont inédites. Les débats actuels n'éclatent

\section{Auteur correspondant : michel.damian@upmf-grenoble.fr}

Michel Damian est professeur émérite à l'Université de Grenoble, où il enseignait notamment l'économie de l'environnement et du développement soutenable à la Faculté d'économie. Il a été directeur du département Gestion de l'environnement à l'Université de la francophonie Senghor d'Alexandrie. Ses travaux récents portent sur l'économie du changement climatique. 
cependant pas dans un ciel serein : ils ont été préparés par trois décennies de conflits environnementaux et une liste déjà longue de catastrophes, ainsi que par les débats entre scientifiques sur la théorie de l'hiver nucléaire et par les récentes négociations américano-soviétiques sur les armes nucléaires. À l'évidence aussi, les mécanismes de l'économie mondiale ont conduit à créer une interdépendance entre les différents écosystèmes de la planète. Ce qui frappe aujourd'hui, c'est l'ampleur des signaux d'alarme. Il est fini le temps où les risques restaient confinés au fond de la mine ou derrière les murs de l'usine. Nous sommes à un tournant. La mise en perspective historique des problèmes globaux confère à ceux-ci une épaisseur singulière. Il ne s'agit pas d'une animation de surface, mais bien d'une mutation dans les consciences, les solidarités et les conflits.

Ce serait pourtant une approche réductrice, et une erreur, de se focaliser sur les seuls grands périls écologiques de l'heure, comme le réchauffement du globe ou l'appauvrissement de la couche d'ozone. Contrairement à la vision de la période récente, le problème ne se limite pas, ne se limite plus, à la seule question des relations de l'homme avec son environnement. Les événements du Golfe, l'actuelle crise militaro-pétrolière, viennent rappeler, en un violent retour de bâton de l'histoire, que les ressources énergétiques ne sont nullement en dehors des problèmes dits globaux. Ici une chose est sûre, il ne suffira pas, en bon scientifique et expert, de modéliser et gérer pour inventer et conserver un avenir viable à la planète.

Dit autrement, il faudra bien en arriver à une gestion globale, moins inégalitaire, moins désordonnée, moins destructrice de la planète et de l'ensemble de ses ressources. C'est assurément notre modèle de développement, mais aussi les multiples égoïsmes nationaux et la question même de la souveraineté nationale qui sont en première ligne. Sur ce terrain, François Perroux, alors qu'il traitait en 1952 de la politique de nationalisation du pétrole en Iran, avait posé quelques jalons qui sont plus que jamais d'actualité : « ni la logique capitaliste pure, ni la logique nationale pure ne peuvent être poussées jusqu'à leur terme ou employées seules pour résoudre un débat d'économie collective et plurinationale. [...] l'organisation collective et supranationale des ressources naturelles s'impose comme une nécessité. » (Perroux, 1952, p. 161 et 168, souligné par l'auteur). Quatre décennies plus tard, la gestion du système Terre - de l'énergie comme des autres ressources - reste à inventer. Les temps présents en montrent l'urgence.

Les lignes qui suivent tentent de poser quelques repères pour une histoire, une généalogie du planétaire, une première pierre pour démêler des racines complexes et enchevêtrées, en situant quelques enjeux assurément durables.

\section{Aux origines, l'atome}

L'énergie nucléaire, ce saut dans le développement des forces productives-destructives, marque une rupture. Longtemps avant la catastrophe de Tchernobyl en 1986.

Dès le printemps 1944, le danois Niels Bohr, qui constitua autour de lui une école fameuse de physique théorique, proposait à Franklin D. Roosevelt et Winston Churchill, mais sans succès, $d$ 'informer le gouvernement soviétique du programme atomique anglo-américain; l'objectif était de parvenir à un accord international sur l'utilisation et le contrôle de l'énergie nucléaire. Dans la même ligne, au début de l'année 1945, des physiciens américains de l'Université de Chicago, dans un rapport remis au ministre de la Guerre, déconseillaient l'utilisation de la bombe contre le Japon et prônaient un contrôle international de l'atome.

$\mathrm{Au}$ sortir de la guerre, le projet est repris par le gouvernement américain. Son plan de désarmement et de contrôle, dit plan Baruch, est présenté le 13 juin 1946, lors de la première réunion de la Commission de l'énergie atomique des Nations unies. Les États-Unis proposaient le transfert des connaissances, des matériaux et des usines dangereuses à une autorité atomique internationale, celle-ci devant être créée et gérée par les pays membres de l'Organisation des Nations unies. L'industrie nucléaire serait donc soustraite à la compétence des États.

Un point essentiel est à retenir de cette période : le nucléaire appelle dès $1^{\prime}$ origine un contrôle, sinon un pouvoir supranational et un embryon d'État mondial. L'autorité atomique proposée était "en somme, un échantillon de gouvernement mondial dans une affaire de portée mondiale. ", a pu écrire Bertrand Goldschmidt (1967, p. 130). À la faillite des négociations entre les États-Unis et l'Union soviétique - les puissances occidentales auraient détenu la majorité des voix au sein de l'autorité atomique -, va succéder la mobilisation de citoyens pour le désarmement et pour la paix. Avec particulièrement, l'appel de Stockholm, lancé en mars 1950 par le Mouvement mondial des partisans de la paix, à l'initiative du prix Nobel Frédéric Joliot-Curie, pour exiger l'interdiction de l'arme atomique et l'établissement d'un contrôle international pour garantir cette interdiction.

Il faudra plus de trois décennies pour parvenir, en 1986-1987, à un premier démantèlement, modeste, de missiles soviétiques et américains à moyenne et courte portée. Ce n'est pas encore la liquidation de l'arme nucléaire, loin de là. Mais c'est un premier pas. Sur le plan de l'atome civil, Tchernobyl est venu rappeler que, là aussi, la politique nationale devait se fondre dans une 
Encadré 1. La question des relations entre environnement et développement en 2015

La thèse de « la pauvreté comme plus grand pollueur » a été énoncée par Indira Gandhi depuis la tribune de la conférence de Stockholm le 14 juin 1972: "Are not poverty and need the greatest polluters? [...] The environment cannot be improved in conditions of poverty. Nor can poverty be eradicated without the use of science and technology. [...] The rich countries may look upon development as the cause of environmental destruction, but to us it is one of the primary means of improving the environment for living. » (Indira Gandhi, Man and environment. Discours à la plenary session of United Nations Conference on Human Environment, Stockholm, June 14, 1972). Ce discours marquant a établi une orthodoxie, en accord avec le point de vue de tous les pays du tiers-monde sur les relations entre environnement et développement ( $c f$. Mukund Govind Rajan, Global environmental politics: India and the North-South politics of global environmental issues, Delhi, Oxford University Press, 1997, p. 25 et 29). Les choses seraient-elles en train de changer? Début mars 2014, Li Keqiang, le premier ministre chinois, déclarait, devant 3000 personnes et en direct à la télévision d'État : «We will resolutely declare war against pollution as we declared war against poverty ».

gestion plus collective, voire dans un contrôle mondial. C'est Andreï Sakharov lui-même, le père de la bombe $\mathrm{H}$ soviétique, et prix Nobel de la paix 1975, qui défendait la thèse en juin 1987 : l'énergie nucléaire, affirmait-il, de par sa nature, doit échapper à la seule autorité des États. Comme si la situation inédite née avec l'atome ne pouvait appeler qu'une solution elle aussi inédite : à problème global, gestion planétaire ? Ce n'est pas qu'une vue de l'esprit ou un leurre. Des formes limitées émergent, certes avec de rudes oppositions d'intérêts : pour le contrôle de l'énergie nucléaire depuis la décennie 1950 ; mais aussi pour l'Antarctique, les océans et fonds marins, l'espace extra-atmosphérique, y compris la Lune, tous déclarés "patrimoine commun de l'humanité ».

\section{Le « vaisseau spatial Terre »}

En 1948, Fairfield Osborn, président de la Société zoologique de New York, publiait le premier best-seller mondial sur la destruction des ressources naturelles. Il y avait pour lui une autre menace que la guerre atomique, celle de la guerre contre la nature : "si nous continuons à faire fi de la nature et de ses principes, les jours de notre civilisation sont dès maintenant comptés. » (Osborn, 1949, p. 14).

Il faudra cependant attendre la décennie 1950 pour la première prise de conscience de l'environnement et de son importance pour la vie humaine. Les retombées radioactives d'origine militaire, avec la présence de strontium 90 dans toutes les parties du monde, en furent le révélateur, dès les années 1953-1954. «Auparavant, écrit le biologiste Barry Commoner, de même que la plupart d'entre nous, je considérais l'eau, l'air et le sol et notre environnement naturel comme plus ou moins immuables et définitivement acquis. » (Commoner, 1972, p. 49). Mais les ressources nécessaires à la vie sont fragiles et limitées, ce sera un des thèmes-phares des décennies qui vont suivre.

Au milieu des années 1960, Kenneth Boulding va devenir célèbre, hors son milieu d'économistes et de sociologues, pour avoir formulé le premier, en 1966, les contraintes d'un monde fini : «la Terre n'est plus qu'un simple vaisseau spatial dépourvu de tout réservoir illimité, que ce soit pour l'extraction ou pour la pollution. » (Boulding, 1966, p. 9, traduction de l'auteur). L'image du « vaisseau spatial Terre » va par la suite faire florès, portée par la critique environnementaliste, avec quelques best-sellers mondiaux sur la pollution, les coûts de la croissance, la population et les ressources (dont le livre phare, Halte à la croissance? commandé par le Club de Rome au MIT et publié sous la direction de Dennis Meadows).

La conférence de Stockholm sur l'environnement humain, organisée par l'Unesco à la demande des Nations unies en 1972, sera l'événement-choc. Le titre du rapport préparatoire - Only one Earth (Ward et Dubos, 1972) - était emblématique. À côté de discours sur la mondialisation des problèmes d'environnement et l'unité de la planète, les divisions et conflits ne seront pas gommés - le sous-développement et la misère ne sont-ils pas les plus grands pollueurs, lançaient les représentants des pays du tiers-monde (Encadré 1) ? La dominance restera néanmoins universaliste: «En pratique, lit-on dans la déclaration finale de la conférence, les problèmes du vaisseau spatial Terre, qui affectent l'humanité tout entière, doivent être abordés sous l'angle mondial. [...] Au moment où nous entrons dans la phase mondiale de l'évolution sociale, il apparaît que chacun de nous a deux pays - le sien propre et la planète Terre. » (Dubos, 1972, p. 22). Suivront des recommandations à l'Organisation des Nations unies, puis des programmes variés menés par les différentes instances et agences de celle-ci. L'essentiel de ces années est en même temps dans les luttes environnementalistes et dans les mouvements de citoyens, qui ont marqué la décennie 1970 aux États-Unis et en Europe. Au seuil des années 1980, ces luttes vont pourtant en partie retomber. 


\section{Pour les militaires et les politiques, une nouvelle représentation du monde}

La reprise des thèmes environnementalistes et de la finitude de la Terre est toute récente. Le nouveau discours sur les risques sort aussi de trois décennies de développement des systèmes militaires et spatiaux (Pignon, 1985, 1989).

L'accumulation de têtes nucléaires stratégiques depuis la décennie 1950 a conduit à une surcapacité à tuer inutilisable dans les faits, avec une dissémination des dépôts d'armes tant en Amérique du Nord qu'en Europe de l'Est et de l'Ouest. Dans le même temps, plus de 2000 satellites ont été mis en orbite autour de la Terre par les États-Unis et l'Union soviétique, dont certains, militaires d'observation, distinguent des détails bien inférieurs au mètre. Pour les experts et stratèges, une identité géopolitique mondiale s'est progressivement constituée : l'ensemble nucléaire et spatial a rendu évident que la Terre est un système fini.

Le glissement des questions militaires aux thèmes environnementaux, mais toujours avec une préoccupation planétaire, remonte à la première moitié de la décennie 1980. Le 23 mars 1983, lorsque le président Reagan lance l'Initiative de défense stratégique (IDS) - « la Guerre des étoiles » -, il s'agit explicitement d'une tentative de restauration de l'hégémonie américaine en traitant la Terre globalement. Mais, paradoxalement, le projet IDS va mettre à mal le dogme de l'invulnérabilité : un bouclier spatial parfait contre les armes atomiques est un non-sens. D'où une première connexion avec le discours pacifiste, avec une prise en compte par le gouvernement américain des propositions soviétiques de réduction de l'arsenal mondial.

Le 15 janvier 1986, Mikhail Gorbatchev répond à Ronald Reagan : non au bouclier spatial mais oui à la destruction d'armes nucléaires. Dans les mois qui suivent, la catastrophe de Tchernobyl, en avril, révèle l'importance des effets secondaires de l'arme atomique, dont l'accident préfigure sur une toute petite échelle les conséquences d'une seule explosion. Les dirigeants soviétiques viennent de recevoir un avertissement : leur technologie n'est pas à toute épreuve.

Dans le même temps, cette double prise de conscience, américaine et soviétique, a été portée par le débat sur la théorie de 1 '« hiver nucléaire »: la catastrophe climatique et écologique engendrée par les conséquences sur l'environnement d'une guerre atomique, théorie étudiée tout d'abord par des scientifiques suédois puis américains. Les Soviétiques vont aussi publier leurs propres simulations et vont symétriquement promouvoir ce débat, en s'adressant à l'opinion publique occidentale dans le contexte des mouvements pacifistes du début de la décennie 1980. Mais les scientifiques soviétiques s'adressaient aussi à leurs dirigeants, pour les convaincre de réorienter leur stratégie nucléaire militaire.

La réorientation des stratégies américaines et soviétiques est aujourd'hui sensible. Depuis décembre 1987, la signature des accords de Washington sur la destruction de missiles de portée intermédiaire marque une volonté nouvelle: réduire les forces en présence. Les relations stratégiques Est-Ouest peuvent être pensées autrement qu'en termes de dissuasion. Pour les militaires et les politiques, il s'agit très certainement d'une nouvelle représentation du monde.

\section{Le changement climatique global}

Le débat sur l'effet de serre n'est sorti des cercles spécialisés qu'au début de la décennie 1980, pour devenir un grand thème de société, intimement lié aux problèmes écologiques et énergétiques du développement (Grinevald, 1990). Cette métamorphose dans le débat n'est pas simple à expliquer.

Parfois depuis longtemps, en silence, loin des médias, des scientifiques s'intéressaient au $\mathrm{CO}_{2}$ et à l'ozone. La mesure systématique et continue du $\mathrm{CO}_{2}$ dans l'atmosphère à l'échelle du globe démarre en 1957-1958, à la suite de l'Année géophysique internationale. Pour l'ozone atmosphérique, les premières études remontent à la fin des années 1920, mais il faudra cependant attendre 1981 pour que soit effectuée la première évaluation de l'état de la couche d'ozone stratosphérique. La National Aeronautics and Space Administration américaine (NASA) met alors en évidence, et pour la première fois, sa diminution au-dessus de l'Antarctique. Mais ce ne sont pas les révélations des scientifiques, à elles seules, qui permettent de comprendre le surgissement tout à fait récent des grands risques planétaires. Les protagonistes de l'IGBP (International Geosphere-Biosphere Programme), vaste programme d'étude du changement global lancé en 1986 par l'ICSU (International Council for Science), ont jusqu'à présent opposé « un non ferme et poli » aux sciences sociales (Burton et Timmerman, 1989 , p. 340). Celles-ci ne seraient pourtant pas de trop, tant pour éclairer les causes humaines et les dimensions sociales des changements de l'environnement planétaire, que pour débrouiller l'explicite et ce qui l'est moins dans les origines et la genèse du programme IGBP. Il faut en effet fortement noter que la prise de conscience et la médiatisation actuelles sont au croisement de préoccupations et de discours scientifiques, géopolitiques et environnementalistes assez différents.

Il y a tout d'abord, et à l'évidence, les constats alarmants. Dans la prolifération des publications et conférences scientifiques, il y a des événements-signes, 
comme des jalons dans la montée des périls. Voici les plus marquants.

Ozone: en mai 1985, publication dans Nature de l'article de Joe Farman, du British Antarctic Survey, sur le trou d'ozone antarctique. La genèse des travaux et la montée des inquiétudes sur ce thème, prégnant depuis le début de la décennie 1970, ont été excellemment présentées par Jonathan Schell (1982). Celui-ci montre tout à fait l'intrication, dans les questionnements successifs, des interrogations des scientifiques, des critiques militantes, des systèmes de valeur, de l'idéologie. C'est une histoire pleinement sociale.

$\mathrm{CO}_{2}$ et effet de serre : en mai 1985, se tient à Villach, Autriche, une conférence climatique devenue célèbre: les participants scientifiques concluent qu'il est aujourd'hui certain que le climat se réchauffe. Peu après cette conférence, le Programme des Nations unies pour l'environnement (PNUE) publie, avec une large diffusion, le premier de ses mini-dossiers sur l'environnement. Son titre, "L'atmosphère en mutation". Les dossiers sur l'environnement, écrit alors le PNUE, sont publiés « pour éveiller l'attention du public sur les questions écologiques globales » (PNUE, 1986a, 1986b).

Ces travaux, qui portent tous sur l'atmosphère et les risques climatiques, sont, semble-t-il, et bien sûr pour partie seulement, un effet indirect des recherches menées depuis le tout début de la décennie 1980 sur la théorie de l' « hiver nucléaire ». La question de l'habitabilité de la planète était étudiée aux États-Unis depuis les années 1970, dans un climat le plus souvent polémique. Mais c'est bien lors des recherches sur $l^{\prime}$ « hiver nucléaire » que furent élaborés les premiers grands modèles permettant de simuler sur ordinateurs les changements climatiques globaux. C'était en quelque sorte l'une des premières évaluations des différentes composantes du système Terre. Et ce sont ces études qui ont aussi alarmé sur les conséquences de la destruction de l'ozone et de l'augmentation de la teneur en $\mathrm{CO}_{2}$ dans l'atmosphère. Lors de la grande conférence sur «Le monde après une guerre nucléaire ", tenue à Washington en octobre 1983 - avec plus de 500 participants, et au cours de laquelle communiquèrent par satellite, et pour la première fois en direct à la télévision, savants américains et russes -, son président concluait de la sorte : «Cet échange de vue pourrait bien être considéré dans les années à venir et à juste titre, comme un tournant décisif de l'histoire de l'humanité » (sur la théorie de $l^{\prime}$ « hiver nucléaire », $c f$., par exemple, les articles publiés dans La Recherche: P. Langley-Danysz, «The year after : les effets biologiques d'une guerre nucléaire", avril 1984 ; A. Berger, «L'hiver nucléaire », juilletaoût 1986). Ces lignes demanderaient à être confirmées (ou infirmées). Mais, à titre d'hypothèse assez solide, il semble bien que le regard nouveau sur l'atmosphère soit pour partie un effet à retardement de « cette effrayante masse de fumée noire ", dont parlait le colonel Tibbets, le pilote de la surperforteresse qui emporta la première bombe atomique au-dessus du Japon (Le Monde, 1945). À tout le moins, et même si les interrogations et travaux sur l'effet de serre remontent bien avant la décennie 1980, les modèles aujourd'hui utilisés doivent quelque chose à ceux mobilisés lors des travaux sur la théorie de l' « hiver nucléaire ». L'histoire complexe de cette nouvelle sensibilité planétaire - les transformations de l'atmosphère comme grand débat de société - reste cependant à écrire (Encadré 2).

\section{La mobilisation pour la planète, des motivations enchevêtrées}

Le vent nouveau est aussi venu de l'Est. Tout au long de la décennie 1970 en Union soviétique, différents écrits, dont ceux publiés dans la revue Les questions de philosophie, tentaient de réfléchir, de répondre notamment aux travaux du Club de Rome, mais aussi de réactiver la réflexion sur les études menées dans les années 1920 par Vladimir Vernadsky sur la biosphère. La révolution dans la dialectique soviétique est cependant toute récente, elle ne remonte qu'au milieu de la décennie 1980. Depuis le XXVII ${ }^{\mathrm{e}}$ congrès du Parti communiste, tenu en février 1986, Mikhail Gorbatchev martèle les thèmes des problèmes environnementaux - «des contradictions globales touchant les fondements mêmes de l'existence de la civilisation »-, de l'interdépendance des États, de l'économie mondiale unique, des intérêts de l'humanité : « se forme un monde contradictoire mais interdépendant, constituant pour beaucoup un tout » (Gorbatchev, 1986, p. 26 et 27). En décembre 1988, à la tribune de l'Organisation des Nations unies, il en appelle à « rechercher en commun la voie vers la suprématie d'une idée universelle, [...] vers la préservation de la vitalité de la civilisation, peut-être unique dans l'univers. » (Gorbatchev, 1988, p. 6). Cette rhétorique - «La Terre, notre maison commune » - était certes à différents usages : elle visait aussi à transformer les problèmes écologiques en atout international (Damian, 1990). En réponse à l'IDS américaine, qui traitait la Terre globalement, les Soviétiques vont proposer de redéployer l'effort scientifique et technologique dans le cadre du désarmement vers, notamment, une investigation et une maîtrise scientifique du système Terre. Le numéro de Time du 2 janvier 1989, largement célébré, avec la planète emballée en couverture - Planet of the Year: Endangered Earth-, est un reflet des nouveaux discours et des nouvelles peurs.

Mais les idées, fussent-elles celles des périls qui menacent la planète, ne font pas leur chemin toute seule. Pour cela, il faut aussi l'activisme des mouvements environnementalistes, des organisations non 
Encadré 2. Des travaux récents (2012-2014) sur les relations entre les scientifiques du nucléaire et du climat

Des connexions et filiations entre laboratoires et scientifiques du nucléaire et du climat sont repérables depuis la décennie 1950, pour l'étude de la concentration de l'atmosphère en radiocarbone (carbone 14), l'étude du cycle du carbone et les premiers modèles climatiques globaux, les travaux sur la théorie de l'hiver nucléaire et ses enjeux planétaires et, enfin, pour l'étude de la concentration de l'atmosphère en $\mathrm{CO}_{2}$ et les recherches sur le changement climatique. Sur ces points, $c f$. le travail excellemment fouillé et précis de Paul N. Edwards en 2012 (« Entangled histories: Climate science and nuclear weapons research », Bulletin of the Atomic Scientists, 68, 4, 28-40). Le célèbre article de Roger Revelle et Hans Suess, publié en 1957, "Carbon dioxide exchange between the atmosphere and ocean and the question of an increase of atmospheric $\mathrm{CO}_{2}$ during the past decades » (Tellus, 9, 18-27), aujourd'hui considéré comme ayant été « the opening shot in the global warming debates » ( $c f$. Weart, S., 2008. The discovery of Global Warming, Harvard University Press), est un produit explicite de ce contexte et de ces intersections. Roger Revelle, océanographe de formation, a longtemps étudié les effets sur l'atmosphère des sous-produits des tests atomiques américains (il avait notamment dirigé, en 1946, une mission d'étude scientifique sur les explosions nucléaires réalisées sur l'atoll de Bikini). Quant à Hans Suess, physicien nucléaire de formation, il avait travaillé au sein du programme atomique allemand durant la Seconde Guerre mondiale. La réorientation d'une partie des laboratoires et scientifiques du nucléaire sera plus nette à partir de la seconde moitié de la décennie 1980 : "Meanwhile, with the Cold War over and research on nuclear weapons in decline, the national laboratories faced a quandary: What would justify their continued existence? Among the labs' greatest assets were their many powerful supercomputers, their expertise in numerical modeling of fluid dynamics, and their skills in managing very large data sets. Many of the labs used these capabilities to refocus their work on environmental problems, particularly those that could make use of advanced computational techniques. Climate change fit this profile perfectly. » (Edwards, op. cit., 2012, 36-37). L'historien Josh Howe, dans un ouvrage récent sur la politique climatique américaine de 1957 à 1997 (Behind the curve: Science and the politics of global warming, Seattle, University of Washington Press, 2014), analyse finement, avec des thèses fortes, le multilatéralisme dominé par la science, le rôle de la guerre froide dans les orientations des travaux des scientifiques, ainsi que celui de la controverse sur " l'hiver nucléaire ", qui a forgé de nouvelles et durables relations entre scientifiques du climat et activistes de l'environnement (voir la présentation et discussion de l'ouvrage par Mike Hulme, 2014, « Behind the curve: Science and the politics of global warming ", Climatic Change, 126, 3-4, 273-278).

gouvernementales, de tous les groupes qui, sur le terrain, contrent l'argumentation et l'action des forces qui détruisent et la nature et les hommes. Sur un plan plus institutionnel, le rapport Brundtland, Our common future (Notre futur à tous, publié en 1987 par la Commission mondiale sur l'environnement et le développement) - et quelles que soient par ailleurs les limites de celui-ci (Beaud, 1989) -, va donner un nouvel élan au thème du devenir collectif, du "développement durable». Fin 1990, ce rapport avait déjà été publié en 25 langues (la seule édition en français est québécoise), et 147 Working Partners, dont la Banque mondiale, étaient alors associés de par le monde au Centre for Our Common Future de Genève (Brundtland Bulletin, 1990). Venu des décennies précédentes, mais aussi de mobilisations institutionnelles et militantes plus récentes, il y a ainsi un foisonnement de réseaux, de mouvements, d'actions: de la vague libérale, semble bien émerger une configuration nouvelle, peut-être porteuse de valeurs plus universelles. Et notamment aux États-Unis : 4 millions d'adhérents aux mouvements environnementalistes en 1980, mais environ 10 millions en 1989 (The Washington Post National Weekly Edition, 1990, p. 34).

\section{L'universel, les silences d'une mutation}

L'effet de serre, le trou dans la couche d'ozone, les "pluies acides ", les menaces très réelles et très immédiates de la pollution des eaux, de la basse atmosphère et des sols, Tchernobyl, mais aussi les questions du désarmement et du développement, du partage et de la gestion des ressources communes, autant d'enseignements, d'interrogations ou d'événements à divers titres terriblement "éducatifs». La notion de risque acquiert une dimension nouvelle. Ces problèmes globaux donnent force aux thèmes de "l'intérêt de l'humanité tout entière " et de "la suprématie d'une idée universelle». Mais qu'est-ce que l'universel ? L'universel n'a aujourd'hui qu'un seul statut, celui du marché mondial capitaliste. On peut alors sourire du discours actuel, apparemment fort consensuel sur le marché comme meilleur instrument pour l'allocation des ressources. La réalité demeure, on le sait, forte de conflits d'intérêts, bien loin des « valeurs universelles » et du « village planétaire ».

Sur le terrain de l'environnement, les seuls débats sur les grands périls pourraient d'ailleurs être ravalés rapidement au second rang. Les divergences actuelles 
entre le Nord et le Sud pour la préparation de la conférence des Nations unies sur l'environnement et le développement, qui se tiendra en juin 1992 au Brésil, en donnent un avant-goût: pour les gouvernements du Sud, il ne suffit pas de placer les grands problèmes écologiques de l'heure au centre des débats, il faut prioritairement insister sur les causes de la détérioration de l'environnement, comme la dette et les échanges commerciaux. S'il n'y a qu'une seule Terre, il y a au moins «deux Mondes» (R.S., 1989). Les 137 gouvernements réunis à Genève du 29 octobre au 7 novembre 1990, sous les auspices de l'Organisation météorologique mondiale, pour la seconde conférence mondiale sur le climat, se sont tous engagés à réduire leurs émissions de gaz à effet de serre. Mais la préservation de la planète et de son climat est lourde d'affrontements durables entre nations, entre l'Est et l'Ouest, entre le Nord et le Sud. Comme l'écrit à juste titre Alexandre Kiss, «le "changement global" prévu par les scientifiques risque de ne pas être seulement celui du climat, mais aussi de bien d'autres choses, y compris du système international. » (Kiss, 1990, p. 8).

D'où des interrogations, mais sans qu'aucune réponse autre qu'équivoque puisse être proposée. Est-il possible de gérer, voire de résoudre les problèmes globaux dans le cadre de l'économie de marché ? C'est le défi des décennies à venir. Qui peut aujourd'hui « représenter les intérêts de l'humanité entière», alors que ceux-ci semblent bien mal assurés par la "main invisible » du marché et la course aux profits ? L'universel et l'humanité ? C'est précisément le contenu énigmatique de la $\mathrm{X}^{\mathrm{e}}$ des Thèses sur Feuerbach de Marx, jamais vraiment analysée par les marxistes: «Le point de vue de l'ancien matérialisme est la société civile bourgeoise ; le point de vue du nouveau, la société humaine ou l'humanité sociale». Marx souligne ici la réciprocité de l'humain et du social. Mais la question du nouveau matérialisme est laissée en blanc, et renvoie donc à des terres parfaitement inconnues (Labica, 1987).

La mutation en cours offre donc bien peu de certitudes. Mais il y a déjà des inflexions, plus ou moins sensibles.

1) Dans l'ordre géopolitique, une représentation inédite du monde se dessine, avec un réagencement général des enjeux et des perceptions stratégiques : l'hégémonie et la sécurité vont maintenant se jouer moins dans la parité des forces nucléaires que dans un discours global sur la gestion de la planète Terre.

2) C'est dans la science que semble aujourd'hui se construire l'universel. D'où l'omniprésence des experts et scientifiques, alors que les risques globaux se prêtent, par leur complexité et par des incertitudes impossibles à combler par l'expérimentation, à des polémiques diverses situées sur des plans différents. Ce rôle grandissant des experts et la difficile, voire impossible, association des populations aux débats et décisions, mettent en question les canons traditionnels de la démocratie participative. Comment associer les différentes sociétés aux travaux des experts et aux décisions des « responsables », pour qu'elles ne continuent à subir, impuissantes, une histoire qui leur échappe?

3) Alors que l'argent et l'affairisme triomphe, le productivisme effréné, tant à l'Ouest qu'à l'Est, atteint les limites de son efficacité. Les économistes néoclassiques raffinent les instruments économiques de gestion de l'environnement - analyse coût-avantage, étude du consentement à payer, systèmes de redevances, taxes et échanges de droits d'émissions (Cropper et Oates, 1990) -, mais, quels que soient par ailleurs l'intérêt et l'efficacité de ces instruments, il faudra autre chose que le jeu du marché pour éviter les catastrophes, diminuer les risques, gérer en commun le devenir de la vie.

4) Aucun État, aucune couche sociale, n'a vocation spontanée et exclusive à prendre en charge, à porter les problèmes globaux. De surcroît, la montée multiforme des nationalismes, des revendications identitaires et des divers intégrismes interdit tout angélisme consensuel et rendra extrêmement difficile toute discussion et toute décision collective et supranationale. C'est la difficulté de la période historique actuelle. D'où, peut-être, le rôle actuellement dévolu, dans un assez grand désordre, aux organisations internationales et aux différentes instances des Nations unies.

5) La gestion du système Terre ne pourra se limiter, se réduire au seul « environnement». C'est l'ensemble des ressources, et donc aussi les ressources énergétiques, qu'il faudrait partager et gérer en commun. Tout est à inventer. Pour le pétrole, $66 \%$ des réserves mondiales sont au Moyen-Orient. Hors son occupation militaire, il n'y a pas d'autre solution que de coopérer, négocier un nouveau deal entre le Nord et le Sud. Plus généralement, l'exhortation ancienne de François Perroux borne irrémédiablement, non seulement les décennies à venir, mais aussi le long terme de notre planète : «l'organisation collective et supranationale des ressources naturelles s'impose comme une nécessité » (Perroux, 1952).

Dans la mise en évidence d'inflexions et de changements, il faut certainement se garder de prendre des bruits résiduels pour des signes de rupture: ne pas « confondre, comme nous le rappelle René Char, une animation de frontières avec un horizon révolutionnaire ». Mais la montée, non seulement des préoccupations environnementales à l'échelle planétaire, mais aussi des problèmes dits globaux, annonce certainement un nouvel âge de l'humanité. La grande mutation ne fait que commencer. 


\section{Références}

Beaud, M., 1989. Risques planétaires, environnement et développement. Sur l'émergence d'une notion, Économie et humanisme, 308, 6-15.

Boulding, K.E., 1966. The economics of the coming spaceship Earth, in Jarrett, H. (Ed.), Environmental quality in a growing economy, Baltimore, Johns Hopkins University Press.

Brundtland Bulletin, 1990. The Centre for Our Common Future, Genève, 8.

Burton, I., Timmerman, P., 1989. Les dimensions sociales des changements planétaires: responsabilités et possibilités, Revue internationale des sciences sociales, 121.

Commoner, B., 1972. L'encerclement, Paris, Seuil [Traduit de Commoner, B., 1971. The Closing Circle, New York, Knopf].

Cropper, M.L., Oates, W.E., 1990. Environmental economics: A survey. Discussion Paper QE90-12, Resources for the Future, Washington, January.

Damian, M., 1990. Environnement, problèmes globaux et crise des sociétés de 1'Est, in Énergie internationale 1990-1991, Paris, Economica.

Dubos, R., 1972. Conférence des Nations unies sur l'environnement, Stockholm, 5-16 juin 1972, Déclaration, plan d'action, recommandations, résolutions. Centre de l'information économique et sociale à l'Office européen des Nations unies.

Goldschmidt, B., 1967. Les rivalités atomiques : 1939-1966, Paris, Fayard.

Gorbatchev, M., 1986. Rapport politique du Comité central du PCUS au XXVII congrès du Parti, 25 février 1986, Agence de presse Novosti.

Gorbatchev, M., 1988. Discours de M. Gorbatchev à l'ONU, Actualités soviétiques, 826, 6.

Grinevald J., 1990. L'effet de serre de la biosphère. De la révolution thermo-industrielle à l'écologie globale, Stratégies énergétiques, biosphère $\mathcal{E}$ société, 1.

Kiss, A., 1990. Changement du climat et changement du droit, Transversales science/culture, 1.
Labica, G., 1987. Karl Marx. Les thèses sur Feuerbach, Paris, PUF. Le Monde, 1945, 9 août.

Meadows, D., Meadows, D., Randers, J., Behrens, W.W. III, 1973. Halte à la croissance?, Paris, Fayard [Traduit de Meadows, D., Meadows, D., Randers, J., Behrens, W.W. III, 1972. The Limits to Growth, Universe Books].

Osborn, F., 1949. La planète au pillage, Paris, Payot [Traduit de Osborn, F., 1948. Our Plundered Planet, Little, Brown \& Co.].

Perroux, F., 1952. L'Anglo-Iranian Oil Company et les effets de domination, Économie appliquée, 1.

Pignon, D., 1985. La guerre-monde, Communications, 42, 87-102.

Pignon, D., 1989. De la guerre des étoiles à la finitude de la Terre : les changements dans les perceptions du risque et leurs conséquences géopolitiques. Communication au séminaire Sécurité, risques, insécurité, ministère de l'Équipement et du Logement, des Transports et de la Mer, Paris, 23 janvier.

PNUE, 1986a. L'atmosphère en mutation, Programme des Nations unies sur l'environnement, Dossier sur l'environnement, 1.

PNUE, 1986b. Quand l'effet de serre menace..., Programme des Nations unies sur l'environnement, Nouvelles du PNUE, mars-avril.

R.S., 1989. Une terre, deux mondes, Ecoforum, Nairobi, août.

Schell, J., 1982. Le destin de la Terre, Paris, Albin Michel [Traduit de Schell, J., 1982. The fate of the Earth, New York, Knopf].

The Washington Post National Weekly Edition, 1990, April 9-15.

Time, 1989. Endangered Earth, 133, 1, January 2.

Ward, B., Dubos, R., 1972. Nous n'avons qu'une terre, Paris, Denoël [Traduit de Ward, B., Dubos, R., 1972. Only one Earth, an unofficial report commissioned by the SecretaryGeneral of the United Nations Conference on the Human Environment].

World Commission on Environment and Development, 1987. Our common future [Bruntdland Report], Oxford, Oxford University Press. 